\title{
Clinical trials for COVID-19 should include sex as a variable
}

\author{
Evelyne Bischof, ${ }^{1,2}$ Jeannette Wolfe, ${ }^{3}$ and Sabra L. Klein ${ }^{4,5}$ \\ 'Department of Advanced Biomedical Sciences, Federico II University of Naples, Naples, Italy. ${ }^{2}$ College of Clinical Medicine, Shanghai University of Medicine and Health Sciences, Shanghai, China. \\ ${ }^{3}$ Department of Emergency Medicine, UMass Medical School - Baystate Campus, Springfield, Massachusetts, USA. ${ }^{4}$ W. Harry Feinstone Department of Molecular Microbiology and Immunology and \\ ${ }^{5}$ Department of Biochemistry and Molecular Biology, Johns Hopkins Bloomberg School of Public Health, Baltimore, Maryland, USA.
}

he number of COVID-19 cases appears to be comparable between men and women, but the severity of disease and death is two times greater for men than for women $(1,2)$. History, including the 1918 influenza pandemic, warned us that male biases in COVID-19 could occur. In this Viewpoint, we focus on biological explanations, with a forward look at why clinicians and biomedical researchers should consider sex as a biological variable that will affect treatment outcomes for COVID-19. There is a long history of not analyzing or reporting differences between women and men in the prophylactic or therapeutic treatment of infectious diseases. We seek to reverse this trend and call on investigators developing and testing therapeutic and prophylactic approaches for COVID-19 to design studies that are inclusive of male versus female differences in drug responses, immunotherapies, vaccines, and nonpharmacological interventions.

\section{Disaggregating data by sex}

Initial reports from Wuhan involving more than 40,000 cases showed that men accounted for nearly two-thirds of deaths, with more severe symptoms and reduced recovery rates (1). This report provided the first alarm and set a precedent for other countries to disaggregate their data by sex (Figure 1). As reports emerged from Italy, Spain, France, and the United Kingdom, men have consistently been twice as likely to die from COVID-19 as women, with the Global Health 50/50 initiative providing real-time sex-disaggregated data from most countries worldwide. Although the United States has been less consistent with sex-disaggregated reporting, a recent analysis of the 26 states with more than 2000 COVID-19 cases revealed that 13 of 26 states disaggregated data using sex as a variable. Notably, all 13 states reported that men are twice as likely to die from COVID-19 (2), with published data from New York City revealing male-biased death from COVID-19 in all adults over the age of 20 (3).

Disparities between men and women in outcomes of infectious diseases, including those caused by pathogenic coronaviruses, are not new. During the 2002-2003 SARS outbreak and the ongoing MERS outbreaks, a majority of patients were men, possibly because of predominantly male contact with the animal reservoirs for these coronaviruses $(4,5)$. However, for many viral respiratory tract infections, including but not limited to coronaviruses, the prevalence and severity of infection is greater for men (6). Preclinical studies show that in mice, males are significantly more susceptible to coronavirus infections, as they are less capable of controlling virus replication, exhibit more post-infection pulmonary damage, and have lower immune responses than females (7).

\section{Drug efficacy}

As of this writing, no antiviral agent has been FDA approved as a specific COVID-19 treatment. For those drugs being tested in randomized clinical trials, there has been no explicit consideration of sex biases in efficacy or adverse reactions. This occurs despite studies showing clear and sometimes profound differences in drug treatment responses, including antivirals. Drug trials are typically designed and analyzed without attention toward sex-specific dosages or differential side effects, while they should be considered in both novel and repositioned drugs.

Conflict of interest: The authors have declared that no conflict of interest exists.

Until recently, remdesivir was regarded as promising for COVID-19, but interim published results are disappointing (8). The still unpublished Adaptive COVID-19 Treatment Trial reported a four-day difference in time to recovery (11 days vs. 15 days) for patients on remdesivir, suggesting some benefit (9). Similar outcomes have been seen for favipiravir, lopinavir, and ritonavir, with the latter causing severe side effects (10). Some studies showed that hydroxychloroquine and chloroquine caused a high number of harmful and lethal casualties (11). All trials to date include both men and women, but take a sex-blind approach to the analyses of outcome data, with no governmental guidelines mentioning sex-specific prophylactic or therapeutic recommendations (except for pregnant and postpartum women). The lack of consideration of sex biases in drug efficacy and reactivity inevitably may lead to increased adverse (potentially lethal) reactions. A study of chloroquine treatment in Brazil, in which $24.7 \%$ of the subjects were female, was prematurely ceased because of complications (especially arrhythmias and QTc prolongation, 25\%) and lethality (17\%). No sex disaggregation was provided for either outcome (12), despite the fact that women are known to suffer more from cardiac intricacies. After the initial therapeutic and suggested prophylactic use of hydroxychloroquine in patients with COVID-19 in March, the FDA released a report on April 24, noting severe and lethal outcomes. It remains underreported whether most of these occurred in women.

Ongoing trials on hydroxychloroquine do not include sex-disaggregated comparisons in their objectives. Available preliminary results of studies on remdesivir and favipiravir also lack sex-specific data on adverse reactions or appropriate dose adjustments. As long as drug trials 


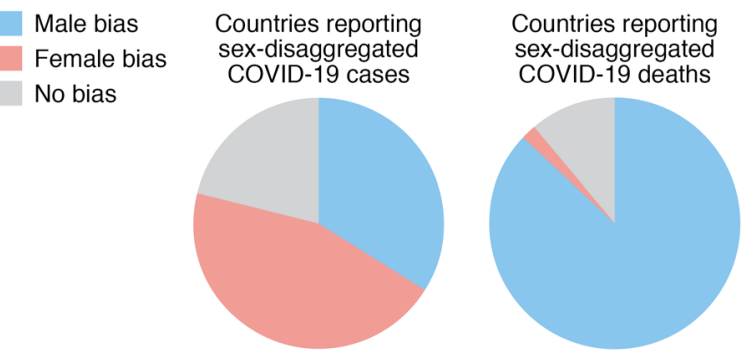

Figure 1. Sex-disaggregated COVID-19 data. The Global Health 50/50 research initiative provides real-time sex-disaggregated data from countries worldwide (accessed May 3, 2020). Based on their analyses of data from 69 countries, $50 \%$ of the countries are sex-disaggregating COVID-19 cases, deaths, or both. For the 44 countries that have sex-disaggregated COVID-19 cases, $34 \%$ report a male bias, $45 \%$ report a female bias, and $21 \%$ report no sex bias (i.e., $50 \% \pm 1 \%$ for each sex). For the 45 countries reporting sex-disaggregated COVID-19 deaths, $87 \%$ report a male bias, $2 \%$ report a female bias, and $11 \%$ report no sex bias (i.e., $50 \% \pm 1 \%$ for each sex).

continue to enroll both men and women but fail to sex-disaggregate outcome data, costly mistakes will continue. Currently, there are over 1000 registered trials on COVID-19, more than half of which include pharmacologic intervention or observation, and seven completed trials. Although the latter were open to both sexes, none reported an objective to balance participants or compared outcomes between men and women.

\section{Immunotherapies}

Immunotherapies for COVID-19 are critical for mitigation of disease and eventual long-term protection against SARS-CoV-2. Because a vaccine is not yet available, other immunotherapies are being evaluated, including the use of convalescent plasma from recovered donors for therapeutic treatment of patients with severe COVID-19 (13). In a small, prospective study in China, convalescent plasma was shown to be safe and effective at improving clinical symptoms and laboratory parameters, with some evidence of reduced viral load (14). In this and other planned studies in the United States, there is no mention of donor sex. For proof of principle, a study in mice revealed that transfer of serum from immune female mice was significantly better at protecting naive mice (both males and females) against influenza than was immune serum from males (15). Immunotherapies that limit characteristics of an inflammatory cascade, e.g., the IL-6 inhibitor tocilizumab, are being tested in studies that enrolled $86 \%$ males, with no indication of comparisons between the sexes (16).
Empirical evidence illustrates that females and males differ in outcomes following the use of therapeutic immunotherapies in autoimmune diseases, infectious diseases, and solid tumors. Females tend to experience more immunotherapyrelated adverse reactions (17). For immunotherapies aimed at stimulating an immune response, e.g., vaccines, females develop stronger responses and may experience greater efficacy than males. In contrast, immunotherapies that repress an immune response, e.g., cytokine or checkpoint inhibitors, are reportedly more efficacious in males $(18,19)$. One-size-fitsall approaches to immunotherapies will not work, and sex/gender may contribute to variable treatment success, including adherence, in clinical settings.

\section{Nonpharmacological management}

Sex differences may influence the necessity or effectiveness of nonpharmaceutical interventions for severe COVID-19. COVID-19 primarily impairs the lungs, and the oxygen requirements of hospitalized patients range from supplementation with a simple nasal cannula to the extreme of extracorporeal membrane oxygenation (ECMO). As early reported mortality rates of intubated COVID-19 were high, discussions arose as to how to best support the respiratory needs of infected patients, especially those who do not physiologically fit the typical acute respiratory distress syndrome (ARDS) pattern and require different ventilatory approaches. Moreover, there has been an intentional shift to avoid early intubation in moderately hypoxic patients by using a combination of noninvasive ventilatory support devices and patient positioning (20). As there are sex-based differences in lung physiology including airway caliber, lung volumes, diaphragmatic excursion, accessory muscle mechanics, and abdominal fat distribution (21), sex-disaggregated outcome data on different invasive and noninvasive ventilatory modalities are crucial, but have not been included in preliminary studies.

Another rapidly evolving area of interest is the increase in thrombotic/ thromboembolic events in patients with COVID-19 (22). Although the main driver of these events is currently unknown, direct viral effects, secondary hypoxia, liver impairment, or the amplification of more classical risk factors, such as severe inflammatory response and immobility, have been suggested (23). A recent review on this topic reinforced the importance of liberal prophylaxis, suggesting therapeutic anticoagulation of all admitted high-risk COVID-19 patients who lack contraindications and prophylactic anticoagulation in outpatients with mild disease and a history of venous thrombosis or immobility (23). Importantly, sex-disaggregated results have not been reported except for a single sentence in the Helms et al. paper, noting that 24 of the 25 patients with pulmonary emboli were male (22). As both under- and overanticoagulation can carry patient risk, further sex-disaggregated information is desperately needed. This information will become even more important if promising estrogen trials ensue, because exogenous estrogen is known to increase the clotting risk in women and in biological males undergoing gender-affirming hormonal therapy (24).

\section{Conclusions}

In medical research, there is a long history of not analyzing or reporting differences between women and men in the presentation and progression of disease, as well as in the prophylaxis or therapy. This occurs despite growing evidence that sex differences exist in the biochemistry and physiology of every organ system. Sex and gender differences also exist in the presentation and prognosis of diverse diseases. The underappreciation of how biological and even social/cultural male-female differences can serve as treatment modifiers 
is a direct reflection of the antecedent of excluding females from biomedical and clinical studies. We seek to reverse this trend and call to action all investigators developing and testing therapeutic and prophylactic approaches for COVID-19 to design studies that are inclusive of male-female differences in responses to drugs, immunotherapies, vaccines, and nonpharmacological interventions.

\section{Acknowledgments}

We thank Shirley Zou and Xin Zhaochen for graphics assistance. We also acknowledge the Gender and COVID-19 working group for ongoing engagement and discussion. EB would also like to acknowledge the Women's Brain Project for discussions on the topic. SK received support from the NIH's Office of Research on Women's Health (ORWH) and the National Institute on Aging (NIA) Specialized Center of Research Excellence (SCORE) on Sex Differences (U54AG062333).

Address correspondence to: Sabra Klein, Department of Molecular Microbiology and Immunology, Johns Hopkins Bloomberg School of Public Health, 615 N. Wolfe Street, Baltimore, Maryland 21205, USA. Phone: 410.955.8898; Email: sklein2@jhu.edu.

1. Wu Z, McGoogan JM. Characteristics of and important lessons from the coronavirus disease 2019 (COVID-19) outbreak in China: summary of a report of 72314 cases from the Chinese Center for Disease Control and Prevention [published online February 24, 2020]. JAMA. https:// doi.org/10.1001/jama.2020.2648.

2. Klein SL, Dhakal S, Ursin RL, Deshpande S, Sandberg K, Mauvais-Jarvis F. Biological sex impacts COVID-19 outcomes. PLoS Pathog. In press.

3. Richardson S, et al. Presenting characteristics, comorbidities, and outcomes among 5700 patients hospitalized with COVID-19 in the New York City area [published online April 22, 2020]. JAMA. https://doi.org/10.1001/ jama.2020.6775.

4. Alghamdi IG, Hussain II, Almalki SS, Alghamdi MS, Alghamdi MM, El-Sheemy MA. The pattern of Middle East respiratory syndrome coronavirus in Saudi Arabia: a descriptive epidemiological analysis of data from the Saudi Ministry of Health. Int J Gen Med. 2014;7:417-423.

5. Karlberg J, Chong DS, Lai WY. Do men have a higher case fatality rate of severe acute respiratory syndrome than women do? Am JEpidemiol. 2004;159(3):229-231.

6. vom Steeg LG, Klein SL. SeXX matters in infectious disease pathogenesis. PLoS Pathog. 2016;12(2):e1005374.

7. Channappanavar R, Fett C, Mack M, Ten Eyck PP, Meyerholz DK, Perlman S. Sex-based differences in susceptibility to severe acute respiratory syndrome coronavirus infection. JImmunol. 2017;198(10):4046-4053.

8. Wang Y, et al. Remdesivir in adults with severe COVID-19: a randomized, double-blind, placebo-controlled, multicentre trial [published online April 29, 2020]. Lancet. https://doi. org/10.1016/S0140-6736(20)31022-9.

9. National Institute of Allergy and Infectious Diseases. NIH clinical trial shows remdesivir accelerates recovery from advanced COVID19. https://www.niaid.nih.gov/news-events/ nih-clinical-trial-shows-remdesivir-acceleratesrecovery-advanced-covid-19. Updated April 19, 2020. Accessed May 12, 2020.

10. Cao B, et al. A trial of lopinavir-ritonavir in adults hospitalized with severe COVID-19. N Engl J Med. 2020;382(19):1787-1799.

11. Juurlink DN. Safety considerations with chloroquine, hydroxychloroquine and azithromycin in the management of SARS-CoV-2 infection. CMAJ. 2020;192(17):E450-E453.

12. Silva Borba MG, et al. Chloroquine diphosphate in two different dosages as adjunctive therapy of hospitalized patients with severe respiratory syndrome in the context of coronavirus (SARSCoV-2) infection: preliminary safety results of a randomized, double-blinded, phase IIb clinical trial (CloroCovid-19 Study). https://doi.org/10.1 101/2020.04.07.20056424. Posted on medRxiv April 16, 2020.

13. Shen C, et al. Treatment of 5 critically Ill patients with COVID-19 with convalescent plasma [pub- lished online March 27, 2020]. JAMA. https:// doi.org/10.1001/jama.2020.4783.

14. Duan K, et al. Effectiveness of convalescent plasma therapy in severe COVID-19 patients. Proc Natl Acad Sci USA. 2020;117(17):9490-9496.

15. Fink AL, Engle K, Ursin RL, Tang WY, Klein SL. Biological sex affects vaccine efficacy and protection against influenza in mice. Proc Natl Acad Sci USA. 2018;115(49):12477-12482.

16. Xu X, et al. Effective treatment of severe COVID-19 patients with tocilizumab [published online April 29, 2020]. Proc Natl Acad Sci USA. https://doi.org/10.1073/pnas.2005615117.

17. Klein SL, Morgan R. The impact of sex and gender on immunotherapy outcomes. Biol Sex Differ. In press.

18. Jawaheer D, et al. Significance of sex in achieving sustained remission in the consortium of rheumatology researchers of North America cohort of rheumatoid arthritis patients. Arthritis Care Res (Hoboken). 2012;64(12):1811-1818.

19. Grassadonia A, et al. Effect of gender on the outcome of patients receiving immune checkpoint Inhibitors for advanced cancer: a systematic review and meta-analysis of phase III randomized clinical trials. J Clin Med. 2018;7(12):E542.

20. Caputo ND, Strayer RJ, Levitan R. Early self-proning in awake, non-intubated patients in the emergency department: a single ED's experience during the COVID-19 pandemic. Acad Emerg Med. 2020;27(5):375-378.

21. Han MK, et al. Female sex and gender in lung/ sleep health and disease. Increased understanding of basic biological, pathophysiological, and behavioral mechanisms leading to better health for female patients with lung disease. Am J Respir Crit Care Med. 2018;198(7):850-858.

22. Helms J, et al. High risk of thrombosis in patients with severe SARS-CoV-2 infection: a multicenter prospective cohort study [published online May 4,2020]. Intensive Care Med. https://doi. org/10.1007/s00134-020-06062-x.

23. Bikdeli B, et al. COVID-19 and thrombotic or thromboembolic disease: implications for prevention, antithrombotic therapy, and follow-up [published online April 15, 2020]. J Am Coll Cardiol. https://doi.org/10.1016/j. jacc.2020.04.031.

24. Getahun D, et al. Cross-sex hormones and acute cardiovascular events in transgender persons: a cohort study. Ann Intern Med. 2018;169(4):205-213. 